\title{
Ecologie alimentaire du Sitatunga (Tragelaphus speckei, Sclater, 1864) dans les sites Ramsar du Sud-Bénin
}

\author{
F.C. KIDJO $^{1 *}$, B.A. DJOSSA ${ }^{2,3}$, M.G. HOUNGBEDJI ${ }^{1}$, T. LOUGBEGNON ${ }^{3,4}$, \\ J. T. C. CODJIA ${ }^{4}$ et B. SINSIN ${ }^{2}$ \\ ${ }^{I}$ Direction Générale du Centre National de Gestion des Réserves de faune (CENAGREF), Rép. du Bénin. \\ ${ }^{2}$ Laboratoire d'Ecologie Appliquée - FSA/UAC/Rép. du Bénin. \\ ${ }^{3}$ Ecole Nationale Supérieure des Sciences et Techniques Agronomiques de Kétou- ENSTA/UAC/Rép. du Bénin \\ ${ }^{4}$ Faculté des Sciences Agronomiques (FSA), Université d'Abomey-Calavi, Rép. du Bénin. \\ *Auteur correspondant, E-mail : claudekidjo@gmail.com
}

\section{RESUME}

La conservation de la diversité biologique dans les zones humides, érigées en sites Ramsar, nécessite une connaissance approfondie de l'écologie de leurs composants majeurs. Pour ce faire, une meilleure connaissance du régime alimentaire des espèces animales menacées de ces sites Ramsar est d'une impérieuse nécessité. Le Sitatunga apparaît actuellement comme l'antilope la plus menacée de la partie méridionale du Bénin. L'étude a été principalement basée sur l'analyse micrographique des crottes de cette espèce. Il ressort des résultats que 74 espèces végétales regroupées en plusieurs catégories fourragères (légumineuses, graminées et autres) sont consommées par le Sitatunga aussi bien pendant la saison sèche que pendant la saison des pluies. Le régime alimentaire du Sitatunga est diversifié et presque invariable toute l'année. Le Sitatunga apparaît enfin comme un consommateur mixte qui marque une préférence pour les prairies et forêts marécageuses, les îlots de végétation autour des marécages, les champs et les jachères.

(C) 2011 International Formulae Group. All rights reserved.

Mots clés : Espèces menacées, antilope, régime alimentaire, zone humide, Sud-Bénin.

\section{INTRODUCTION}

Le régime alimentaire est un élément important dans l'étude de la biologie de conservation car la disponibilité des ressources alimentaires affecte la dynamique et la viabilité des populations d'espèces animales (Johnson et Sherry, 2001; Moegenburg et Levey, 2003). L'analyse du régime alimentaire permet d'apprécier le degré de recouvrement et des préférences alimentaires de différents ongulés (Hansen et al., 1973). Plusieurs méthodes ont été développées pour inventorier les ressources alimentaires utilisées par les animaux en milieux naturels ou en captivité (Short, 1981). L'étude microscopique des fèces comme outil d'étude du régime alimentaire a été développée pour la première fois dans l'optique d'une étude globale du régime alimentaire des écureuils (Scriurus spp.) par Baumgartner et Martin (1939) puis par d'autres auteurs (Kiley, 1966) sur différentes espèces. Cette méthode indirecte a été, par la suite, utilisée dans le suivi 
écologique de différentes espèces animales par beaucoup d'autres auteurs (Short, 1981; Hanley et al., 1985 ; Maizeret et al., 1986). La nécessité d'étudier l'écologie alimentaire du Sitatunga par la méthode micrographique, objet de la présente étude, se justifie par le fait que cette antilope menacée est devenue si rare et donc difficile à observer qu'un suivi direct au pâturage est devenu presque impossible, encore qu'elle peut aussi pâturer la nuit. Aussi, cette espèce est-elle considérée comme une espèce-phare dont la conservation aura un effet d'entraînement pour beaucoup d'autres espèces inféodées à ces écosystèmes. Mais, cette espèce d'antilope n'a fait jusqu'à présent l'objet d'aucune étude spécifique pour sa conservation au Bénin. La présente étude permet donc de rassembler les connaissances scientifiques nécessaires pour comprendre l'écologie alimentaire du Sitatunga et partant, contribuer à la conservation des zones humides qui sont classées sites Ramsar, habitats de prédilection de cette espèce au Bénin. Il s'agissait surtout dans cette étude de déterminer la composition floristique du régime alimentaire du Sitatunga dans les différents écosystèmes fréquentés et durant les différentes saisons.

\section{MATERIEL ET METHODES}

\section{Milieu d'étude}

Au Bénin les zones humides couvrent une bonne partie de la région méridionale située entre les latitudes $6^{\circ} 15$ et $7^{\circ} 00$ et se subdivisent en deux: le complexe Est et le complexe Ouest (Houndagba et al., 2001).

Le climat de cette région est du type subéquatorial avec deux saisons pluvieuses (mi-mars à juillet; mi-septembre à minovembre) et deux saisons sèches (mi-juillet à mi-septembre ; mi-novembre à mi-mars). Les pluies sont concentrées sur les mois d'avril et de juin. Les moyennes pluviométriques annuelles varient de 1000 à $1500 \mathrm{~mm}$. L'hygrométrie moyenne est toujours supérieure à 60\% (Akoègninou, 1984). La température moyenne annuelle est de $27,5^{\circ} \mathrm{C}$.
Le Sud-Bénin abrite de nos jours une mosaïque de formations végétales naturelles et anthropiques (Adomou, 2005). Les formations anthropiques (champs, jachères et plantations) sont plus étendues du fait de la forte densité des populations humaines. Les principales spéculations vivrières sont le maïs, le manioc, le niébé, les cultures maraîchères et fruitières dont le palmier à huile. Les formations naturelles se répartissent globalement en formations de terres fermes et en formations de zones humides (Adjakidjè et Sokpon, 2001). On distingue: les mangroves à Rhizophora et Avicennia, les forêts marécageuses, les forêts périodiquement et occasionnellement inondées, les savanes marécageuses à Mitragyna inermis et Andropogon gayanus var. squamulatus, les forêts-galeries à Pterocarpus santalinoides et Manilkara multinervis, la végétation herbacée des milieux saumâtres et des lagunes en communication temporaire avec l'océan, composée des prairies à Echinochloa pyramidalis, à Cyclosorus striatus, de fourré à Dissotis segregeta et enfin les formations artificielles des zones humides.

La faune dans les zones humides du Sud-Bénin est encore riche et diversifiée. La faune aviaire est composée de plus de 233 espèces d'oiseaux réparties en 52 familles (Adjakpa, 2001). Les travaux d'inventaires (Hessou et Houngnon, 1999; Kidjo et Guédou, 2001) font état de plus de 16 espèces de mammifères dans les zones humides dont les plus remarquables sont: Hippopotame (Hippopotamus amphibius), Lamentin d'Afrique (Trichechus senegalensis), Sitatunga (Tragelaphus spekei), Guib harnaché (Tragelaphus scriptus), Daman des arbres (Dendrohyrasx dorsalis sylvestris), singe à ventre rouge (Cercopythecus $e$. erytrogaster), le vervet (Cercopythecus eathiops), Colobes noir et blanc de l'Afrique occidentale (Colobus polykomos), les loutres (Lutra maculicollis et Aonyx capensis), Génettes (Genetta pardina et Genetta tigrina), le potamochère (Potamochorus porcus) etc. 
Des quatre bassins hydrographiques dont dispose le Bénin, deux conditionnent le réseau hydrographique du Sud-Bénin, il s'agit de l'Ouémé et du Mono (Figure 1).

La présente étude a été conduite sur les sites Ramsar du Sud-Bénin qui constituent l'aire d'occurrence du Sitatunga.

\section{Choix des sites}

A l'issue d'une étude exploratoire sur les sites Ramsar (complexe Ouest et le complexe Est) les zones où le Sitatunga peut potentiellement être rencontré ont été identifiées et une enquête dans les populations riveraines des zones humides a permis de rapporter des informations sur la présence ou non du Sitatunga. Ainsi, trois grandes zones ont été retenues compte tenu de leur habitat et de la présence actuelle du Sitatunga (Figure 1). Il s'agit:

* des marécages du Sud de la vallée du lac Ahémé dans les communes de Ouidah et de Kpomassè,

* de la vallée de l'Ouémé dans les communes de Sèmè-Kpodji, Zê et de Bonou,

* de la forêt marécageuse de Lokoli dans la commune de Zogbodomè.

Un total de 18 transects dont 6 dans chacune des trois zones définies ci-dessus ont été retenus pour la collecte régulière des données sur la présence et les fréquentations de l'animal d'étude.

\section{Animal d'étude Description}

Le Sitatunga (Tragelaphus spekii Speke) de la famille des bovidés, sous-famille des Tragelaphinae est une antilope dont la couleur de la robe varie au sein d'une même population mais reste généralement d'un rouge sombre chez la femelle et le mâle immature. En mûrissant, la robe des mâles varie graduellement vers un gris chocolat sur un fond brun arborant une crinière et une raie dorsale blanche. Il y a des taches blanches sur le visage, des raies et des taches sur le corps chez les deux sexes bien que le pelage soit globalement obscur. Les sabots sont longs et extrêmement pointus leur permettant de se déplacer dans un milieu marécageux. Le poids moyen corporel est de $87,5 \mathrm{~kg}$ et la hauteur au garrot est d'environ $100 \mathrm{~cm}$ chez le mâle alors qu'elle varie de 75 à $90 \mathrm{~cm}$ chez la femelle (Kingdom, 1997).

\section{Alimentation}

Cette espèce pâture tant dans les zones humides que sur terre ferme. Elle a une préférence pour les plantes en inflorescence. Elle émerge dans la nuit et pâture sur les terres fermes adjacentes à la zone humide qui l'abrite.

La maturité sexuelle se situe autour d'un an, la durée de gestation d'environ 8 mois et la portée est de 1 ou 2 petits.

\section{Habitats et distribution}

Cette espèce est rencontrée dans les formations marécageuses à inondation saisonnière où existent de hautes herbes, des galeries forestières, des mangroves. En savane elle est inféodée à des formations herbeuses dominées par Cyperus spp., Phragmites spp. et Echinochloa spp. Elle évite les plans d'eau dépourvus de végétation. Sa distribution est très vaste en Afrique Centrale, alors qu'en Afrique de l'Ouest, sa présence est seulement ponctuelle, notamment en Gambie, Guinée Conakry, Guinée Bissau, Côte d'Ivoire, Ghana et Nigéria. Au Bénin, cette espèce devenue rare de nos jours est mentionnée dans le Sud du pays au niveau des forêts marécageuses d'Agonvè (Zagnanado), de Zinvié-Kpotomey-Lokoli, de Hozin, de Pahou et dans la forêt classée de la Lama. La fragmentation des habitats, les pertes des milieux humides et les pressions de chasse constituent les menaces qui pèsent sur cette espèce.

\section{Méthodes de collecte de données \\ Principe de la méthode d'analyse micrographique}

La paroi cellulosique externe des cellules épidermiques végétales est recouverte 
d'une couche de polymères d'acides gras insaturés appelée cuticule. Cette cuticule qui protège les cellules végétales résiste très bien à la dégradation chimique, donc aux enzymes digestives (Chapuis, 1980 ; Maizeret et al., 1986). Il y a donc de forte probabilité de retrouver dans les fèces des fragments épidermiques non altérés ou partiellement altérés. Par ailleurs, chaque espèce végétale possède des cellules épidermiques caractérisées par une morphologie particulière: taille, forme, présence ou absence de stomates, agencement des cellules (Planton, 1987). Cette spécificité structurale permet ainsi de déterminer à partir d'un fragment épidermique l'espèce végétale pâturée par comparaison avec des structures épidermiques de référence. Ainsi, deux types de lames à savoir les lames de références et les lames-échantillons sont nécessaires pour une analyse micrographique des crottes.

Inventaire des espèces appétées par le Sitatunga sur la base d'une enquête en vue de la préparation des lames de référence

Sur la base des déclarations des populations locales, les espèces végétales consommées par le Sitatunga ont été listées, collectées et identifiées. Ces espèces ont servi à préparer les lames de référence, lesquelles lames ont ensuite servi de témoins pour identifier les espèces végétales réellement consommées par l'animal lors de l'analyse microscopique des épidermes végétaux (Lazzarini, in Ayégnon, 2004). Ainsi, la composition floristique du régime alimentaire du Sitatunga a été déterminée. Cette analyse a été faite au Laboratoire des Techniques d'Aménagement et Protection de l'Environnement de l'Ecole Polytechnique d'Abomey-Calavi de l'Université d'AbomeyCalavi.

\section{Collecte de crottes pour préparer les lames- échantillons}

Lors de l'inventaire sur la base des déclarations des populations riveraines, des sites d'observation et de collecte des données ont été choisis en tenant compte de la géomorphologie au niveau des différentes formations végétales dans chacune des zones prospectées mais aussi et surtout de l'existence des indices de présence et des rencontres du Sitatunga.

Dans chacun des sites retenus, les pistes ouvertes par l'espèce et les champs de cultures visités par l'espèce (sur la base des indices de présence) ont été parcourus mensuellement suivant des transects linéaires pour collecter des crottes fraîches. Au niveau de chaque transect, en plus des crottes les autres indices de présence tels que les restes de nourriture, les empreintes ainsi que les présences physiques sont rapportés.

\section{> Préparation des lames de référence}

Les fragments de feuilles des espèces végétales récoltées et identifiées sont réhydratées au laboratoire pendant 1 ou 2 min dans de l'eau de javel (solution à $5 \%$ ). Les faces dorsales et ventrales de ces feuilles ont été grattées à l'aide d'une lame de Bistouri et ce, jusqu'à ce que les faces deviennent bien translucides. Les structures morphologiques de l'épiderme des faces dorsales et ventrales étant souvent différentes pour une même espèce végétale, il est indispensable de faire des préparations des deux faces foliaires (Schockert, 1997).

Le fragment végétal gratté et raclé au moyen de la lame de Bistouri est rendu transparent après un bain de 1 à 3 min dans l'eau de javel diluée à 95\%. Les fragments traités sont ensuite montés entre lame et lamelle dans une goutte de Mowiol ou de glycérol. Les lamelles sont ainsi scellées aux lames et les préparations ainsi obtenues à partir de plantes connues sont étiquetées puis photographiées sous microscope.

\section{> Préparation des lames-échantillons}

Dix échantillons de tas de crottes du Sitatunga collectées chaque mois ont été soumis aux traitements préalables à l'analyse micrographique:

- Les crottes ont été écrasées au moyen d'un mortier et un pilon de laboratoire afin qu'elles se délitent plus facilement, 
- Une petite quantité ( $2 \mathrm{~g})$ de ce substrat a été prélevée dans un bêcher en pyrex. On y a ajouté de l'eau de javel diluée à 10\% (20 ml d'eau de javel pour $200 \mathrm{ml}$ d'eau courante). L'ensemble a été porté sur un agitateur magnétique pendant 15 min. Grâce à un tamis à mailles très fines, on a procédé au filtrage de la préparation. Les fines particules de crottes entraînées par l'eau javellisée et qui se sont déposées en formant un culot ont été récupérées et la solution surnageante a été rejetée. Ces particules de crottes ont ensuite été rincées à grande eau, puis laissées égouttées.

Pour chaque échantillon de crotte traité comme décrite ci-dessus, une petite quantité du culot a été étalée sur une lame recouverte de Mowiol ou de glycérol puis scellée avec une lamelle avant d'être observée au microscope à différents grossissements.

Sur chacune des lames-échantillons préparées, on a procédé à une série de quinze lectures. A chaque lecture, on a identifié un et un seul item végétal (élément minimal d'un fragment végétal). Les items végétaux non identifiés ont pu être classés au moins soit dans la catégorie des monocotylédones diverses, soit dans celle des dicotylédones diverses.

\section{Traitement des données collectées}

L'analyse des données a été faite à l'aide de trois paramètres à savoir: la largeur $w$ de l'espace alimentaire, l'indice de diversité de Shannon et l'équitabilité de Pielou.

* l'indice de diversité de Shannon (H'). $\mathbf{H}^{\prime}=-\boldsymbol{\Sigma}$ pi $\log _{2}$ pi avec $\mathrm{Pi}=$ proportion de l'espèce $i$ dans l'espace alimentaire.

$\mathbf{N i} / \mathbf{N}=\mathbf{P i}$ avec Pi : proportion de l'espèce $i$ par rapport au nombre de fragments. $\mathrm{Ni}$ : Nombre de fragments de l'espèce $i, \mathrm{~N}$ : Nombre total de fragments

\section{* Equitabilité de Pielou}

Elle est donnée par la formule: $\mathrm{E}=$ $\mathrm{H}^{\prime} / \log _{2} \mathrm{~S}$ avec E $\in[0,1]$ et $\mathrm{S}$ désignant la diversité totale
Si $0<\mathrm{E}<0,5$ la diversité est faible, Si $0,5<$ $\mathrm{E}<0,7$ la diversité est moyenne, Si E $>0,7$ la diversité est forte.

* la largeur $w$ de l'espace alimentaire (= diversité du régime alimentaire) $\boldsymbol{w}$ = 2H' ' avec H',

$=-\left(\right.$ Pim* $\log _{2}$ Pim - Pid* $\log _{2}$ Pid $)$ avec Pim = Proportion des monocotylédones, Pid = Proportion des dicotylédones, $\boldsymbol{w} \mathbf{C}[\mathbf{1} ; \boldsymbol{i}] \quad i=$ nombre de classe ou de catégories fourragères définies

La nature mathématique de $w$ est bornée par 1 quand toutes les ressources utilisées sont concentrées dans une classe et par la variable $i$ ou le nombre total de classe dans le cas où les ressources utilisées sont équitablement distribuées dans toutes les classes.

Les proportions moyennes des items végétaux obtenus au cours des trois années sont calculées.

\section{RESULTATS}

\section{Pâturages exploités par le Sitatunga}

$\mathrm{Au}$ total 18 transects ont été mensuellement parcourus pour les collectes de données dans les complexes Ouest et Est. Sur la base des indices de présence (crottes et empreintes) et des contacts avec l'animal, les habitats exploités ont été listés et les intensités d'utilisation ont aussi été rapportées (Tableau $1)$.

Les prairies et forêts marécageuses sont les pâturages les plus exploités par le Sitatunga. Mais ce tragélaphiné exploite aussi parfois les galeries forestières, les îlots de végétation autour des marécages, les champs et jachères (Tableau1). Enfin l'intensité de l'exploitation de chacun des pâturages identifiés varie en fonction des saisons (périodes de hautes eaux et de basses eaux).

Diversité floristique du régime alimentaire du Sitatunga dans les zones humides du Sud-Bénin

$\mathrm{Au}$ total, 74 espèces végétales appartenant à 48 familles ont été recensées au 
cours des trois années d'étude. La préférence des espèces végétales par le Sitatunga n'est pas la même. Cette préférence a été identifiée à travers les proportions des items végétaux retrouvés dans les crottes au cours de l'analyse micrographique.

De l'analyse des résultats, il ressort que trois catégories de plantes peuvent être distinguées dans la liste des espèces consommées par le Sitatunga. Il s'agit de :

Catégorie 1: espèces faiblement appétées, Catégorie 2 : espèces moyennement appétées, Catégorie 3 : espèces prioritairement appétées

$>$ La première catégorie, constituée de 41 espèces représente approximativement $57 \%$ des espèces appétées (Tableau 2).

Les espèces constituant la première catégorie sont consommées par le Sitatunga mais seraient faiblement appréciées par ce dernier. Il s'agit des espèces dont les proportions se situent dans l'intervalle $[0,001 ; 0,01]$. En ce qui concerne les formes végétatives, les herbes dominent avec $40 \%$ des items suivis des arbustes (30\%), des arbres $(12 \%)$ et des lianes $(18 \%)$.

$>$ La deuxième catégorie, constituée de 29 espèces, représente approximativement $38 \%$ des espèces appétées et comporte les espèces qui peuvent être considérées comme moyennement appétées (Tableau 3).

Il s'agit de 29 espèces végétales appétées parmi lesquelles Opilia celtidifolia, Chassalia kolly, Mimosa pigra, Ipomoea aquatica et Mallotus oppositifolius viennent en tête par ordre d'importance. En termes de forme végétative, les herbes dominent avec $52 \%$ des items suivis des arbustes $(31 \%)$, des arbres (7\%) et des lianes (10\%).

$>$ La troisième catégorie, constituée de 4 espèces, représente approximativement $5 \%$ des espèces appétées et comporte les espèces qui peuvent être considérées comme prioritairement appétées (Tableau 4).
De ce tableau, il ressort que quatre espèces végétales sont préférentiellement consommées par le Sitatunga et constituent les espèces les mieux appréciées et les mieux consommées dans les zones humides du SudBénin avec une proportion variant dans l'intervalle [0,0613; 0,118].

En cas d'une éventuelle sélection ces quatre espèces seraient les plus privilégiées par le Sitatunga.

De l'analyse de ces trois tableaux précédents, il ressort que le Sitatunga n'a pas la même préférence pour les espèces recensées et qui constituent son régime alimentaire.

\section{Variabilité de l'espace alimentaire du Sitatunga}

Les proportions des items végétaux dénombrés sur 50 lames préparées à partir des crottes du Sitatunga et réparties par classe et catégorie fourragères sont présentées sur la Figure $1 a \& b$.

Le régime alimentaire du Sitatunga est donc assez diversifié et dominé par les espèces ligneuses (dicotylédones).

En considérant les différents groupes fourragers (monocotylédone, dicotylédones, légumineuses, graminées et autre catégorie fourragère) et en les combinant deux à deux pour le calcul de la valeur $w$ de l'espace alimentaire $(w=1,9) ; w \in[1,2]$ et tend vers 2. Cette valeur montre que toutes les classes considérées sont exploitées à la fois par le Sitatunga. Ce qui signifie que cette antilope est par conséquent un consommateur mixte.

Les valeurs de l'indice de diversité de Shannon $\mathrm{H}=4,6$ bits et de l'indice de régularité de Pielou $\mathrm{E}=0.9$ calculées montrent que le régime alimentaire du Sitatunga est constitué d'une gamme variée d'espèces végétales. 
F.C. KIDJO et al. / Int. J. Biol. Chem. Sci. 5(2): 603-617, 2011

Tableau 1 : Pâturages exploités par le Sitatunga dans la zone d'étude.

\begin{tabular}{|c|c|c|c|c|c|c|}
\hline & $\begin{array}{l}\text { Indice } \\
\text { de présence }\end{array}$ & Prairie marécageuse & $\begin{array}{c}\text { Forêt } \\
\text { marécageuse }\end{array}$ & Galerie forestière & $\begin{array}{c}\text { Ilots forestiers } \\
\text { autour des } \\
\text { marécages }\end{array}$ & $\begin{array}{c}\text { Champs } \\
\text { et } \\
\text { jachères }\end{array}$ \\
\hline \multirow{3}{*}{$\begin{array}{l}\text { Période de basses } \\
\text { eaux }\end{array}$} & Empreintes & $>20$ & $<20$ & $<15$ & $<5$ & $<5$ \\
\hline & $\%$ Crottes & $47 \%$ & $26 \%$ & $17 \%$ & $11 \%$ & $9 \%$ \\
\hline & Fréquence de rencontre & $51 \%$ & $33 \%$ & $8 \%$ & $8 \%$ & $0 \%$ \\
\hline \multirow{3}{*}{$\begin{array}{l}\text { Période de hautes } \\
\text { eaux }\end{array}$} & Empreintes & $<5$ & $<5$ & $<5$ & $<15$ & $>20$ \\
\hline & $\%$ Crottes & $0 \%$ & $7 \%$ & $8 \%$ & $29 \%$ & $56 \%$ \\
\hline & Fréquence de rencontre & $0 \%$ & $0 \%$ & $0 \%$ & $35 \%$ & $65 \%$ \\
\hline
\end{tabular}

Tableau 2 : Proportions des items végétaux faiblement consommés par le Sitatunga.

\begin{tabular}{lllc}
\hline Espèce & Famille & Forme végétative & Largeur $(\boldsymbol{w})$ \\
\hline Abutilon mauritianum & Malvaceae & Herbe & 0,00657 \\
Alchornea cordifolia & Euphorbiaceae & Herbe & 0,00933 \\
Antiaris toxicaria & Moraceae & Arbre & 0,00304 \\
Blighia sapida & Sapindaceae & Arbre & 0,00125 \\
Bridelia ferruginea & Euphorbiaceae & Arbuste ou petit arbre & 0,0026 \\
Callichilia barteri & Apocynaceae & Arbuste & 0,007 \\
Carissa edulis & Apocynaceae & Arbuste & 0,00115 \\
Cassipourea barteri & Rhizophoraceae & Arbuste ou petit arbre & 0,00276 \\
Chromolaena odorata & Asteraceae & Suffrutex & 0,00946 \\
Clappertonia ficifolia & Tiliaceae & Arbuste & 0,0105 \\
Clerodendrum capitatum & Verbenaceae & Arbuste érigé ou lianescent & 0,00998 \\
Cnestis ferruginea & Connaraceae & Liane ou arbuste & 0,00545
\end{tabular}


F.C. KIDJO et al. / Int. J. Biol. Chem. Sci. 5(2): 603-617, 2011

\begin{tabular}{lllc} 
Cyperus papyrus & Cyperaceae & Herbe vivace à rhizome & 0,00005 \\
Dialium guineense & Leguminosae-Caesalpinioideae & Arbre & 0,00659 \\
Emilia sonchifolia & Asteraceae & Herbe & 0,00565 \\
Enydra fluctuans & Asteraceae & Herbe vivace & 0,00722 \\
Eriocaulon fulvum & Eriocaulaceae & Herbe & 0,00818 \\
Flacourtia flaverscens & Flacourtiaceae & Arbuste ou petit arbre & 0,0039 \\
Heterotis rotundifolia & Melastomataceae & Herbe & 0,00953 \\
Ipomoea involucrata & Convolvulaceae & Herbe annuelle ou vivace & 0,00413 \\
Ipomoea mauritiana & Convolvulaceae & Herbe vivace & 0,00261 \\
Ipomoea rubens & Convolvulaceae & Herbe vivace & 0,00211 \\
Jasminium dichotomum & Oleaceae & Arbuste grimpant & 0,00447 \\
Lygodium mycrophyllum & Schizaeaceae & Liane rampante & 0,0033 \\
Mikania cordata & Asteraceae & Herbe vivace & 0,0107 \\
Morinda lucida & Rubiaceae & Arbre & 0,00115 \\
Mussaenda arcuata & Rubiaceae & Arbuste & 0,00439 \\
Mussaenda issertiana & Rubiaceae & Plante grimpante & 0,00194 \\
Panicum parvifolium & Poaceae & Herbe pérenne & 0,00639 \\
Paropsia guineensis & Passifloraceae & Arbre & 0,00832 \\
Pavetta corymbosa & Rubiaceae & Arbuste ou petit arbre & 0,002 \\
Pentodon pentandrus & Rubiaceae & Herbe annuelle & 0,00693 \\
Polygonum lanigerum & Polygonaceae & Herbe pérenne & 0,00395 \\
Psychotria calva & Rubiaceae & Arbuste & 0,00178 \\
Rhynchospora corymbosa & Cyperaceae & Plante vivace & 0,00713 \\
Sterculia tragacantha & Sterculiaceae & Petit arbre & 0,00755 \\
Stylosanthes erecta & Leguminosae-Papilionoideae & Sous arbuste & 0,00615 \\
Thalia welwitschii & Marantaceae & Herbe en touffe & 0,0038 \\
Tristemma hirtum & Melastomataceae & Herbe ou sous arbrisseau & 0,00797 \\
Uvaria shamae & Annonaceae & Arbuste sarmenteux & 0,00206 \\
Vigna unguiculata & Leguminosae-Papilionoideae & Herbe pérenne & 0,00821 \\
\hline & & &
\end{tabular}


F.C. KIDJO et al. / Int. J. Biol. Chem. Sci. 5(2): 603-617, 2011

Tableau 3 : Proportions des items végétaux moyennement consommés par le Sitatunga.

\begin{tabular}{lllc}
\hline \multicolumn{1}{c}{ Espèce } & \multicolumn{1}{c}{ Famille } & \multicolumn{1}{c}{ Forme végétative } & Largeur $(\boldsymbol{w})$ \\
\hline Acroceras zizanioides & Poaceae & Herbe pérenne & 0,013 \\
Aeschynomene indica & Leguminosae-Papilionoideae & Sous arbuste & 0,0293 \\
Albizia adianthifolia & Leguminosae-Mimosoideae & Arbre & 0,0168 \\
Andropogon gayanus & Poaceae & Herbe & 0,0177 \\
Anthocleista vogelii & Loganiaceae & Arbre & 0,0202 \\
Cassytha filiformis & Lauraceae & Parasite à feuille volubile & 0,0131 \\
Chassalia kolly & Rubiaceae & Arbuste & 0,0384 \\
Croton lobatus & Euphorbiaceae & Herbe & 0,0173 \\
Cyperus articulatus & Cyperaceae & Herbe vivace & 0,0125 \\
Fuirena umbellata & Cyperaceae & Herbe vivace & 0,0201 \\
Ipomea aquatica & Convolvulaceae & Herbe vivace & 0,0357 \\
Ludwigia decurrens & Onagraceae & Herbe & 0,0166 \\
Ludwigia octovalvis & Onagraceae & Herbe & 0,017 \\
Lycopersicon esculentum & Solanaceae & Herbe annuelle & 0,0165 \\
Mallotus oppositifolius & Euphorbiaceae & Arbuste & 0,0332 \\
Manihot esculenta & Euphorbiaceae & Arbuste & 0,0145 \\
Mimosa pigra & Leguminosae-Mimosoideae & Arbuste épineux & 0,0373 \\
Morelia senegalensis & Rubiaceae & Arbuste & 0,015 \\
Opilia celtidifolia & Opiliaceae & Liane & 0,0392 \\
Pauridiantha hirtella & Rubiaceae & Arbrisseau ou arbuste & 0,0193
\end{tabular}


F.C. KIDJO et al. / Int. J. Biol. Chem. Sci. 5(2): 603-617, 2011

\begin{tabular}{llll} 
Phyllaunthus amarus & Euphorbiaceae & Herbe érigée & 0,0201 \\
PouteriaMalacantha alnifolia & Sapotaceae & Arbuste ou petit arbre & 0,014 \\
Raphiostylis beninensis & Icacinaceae & Arbuste grimpant & 0,0202 \\
Ritchiea capparoides & Caricaceae & Arbuste ou petit arbre & 0,0147 \\
Rourea coccinea & Connaraceae & Herbe & 0,0155 \\
Sesanum indicum & Solanaceae & Herbe suffrutescente ou arbuste & 0,0128 \\
Sporobolus pyramidalis & Poaceae & Herbe & 0,0143 \\
Vernonia cinerea & Asteraceae & Herbe & 0,0145 \\
Vigna ambacensis & Leguminosae-Papilionoideae & Herbe & 0,0193 \\
\hline
\end{tabular}

Tableau 4 : Proportions des items végétaux préférentiellement consommés par le Siatunga.

\begin{tabular}{lllc}
\hline Espèce & Famille & Forme végétative & Largeur $(\boldsymbol{w})$ \\
\hline Albizia zygia & Leguminosae-Mimosoideae & Arbre & 0,118 \\
Sorendeia warneckei & Anacardiaceae & Arbuste & 0,074 \\
Lecaniodiscus cupanioides & Sapindaceae & Arbuste ou petit arbre & 0,0613 \\
Cyrtosperma senegale & Araceae & Grande herbe à rhizomes & 0,0704 \\
\hline
\end{tabular}


F.C. KIDJO et al. / Int. J. Biol. Chem. Sci. 5(2): 603-617, 2011

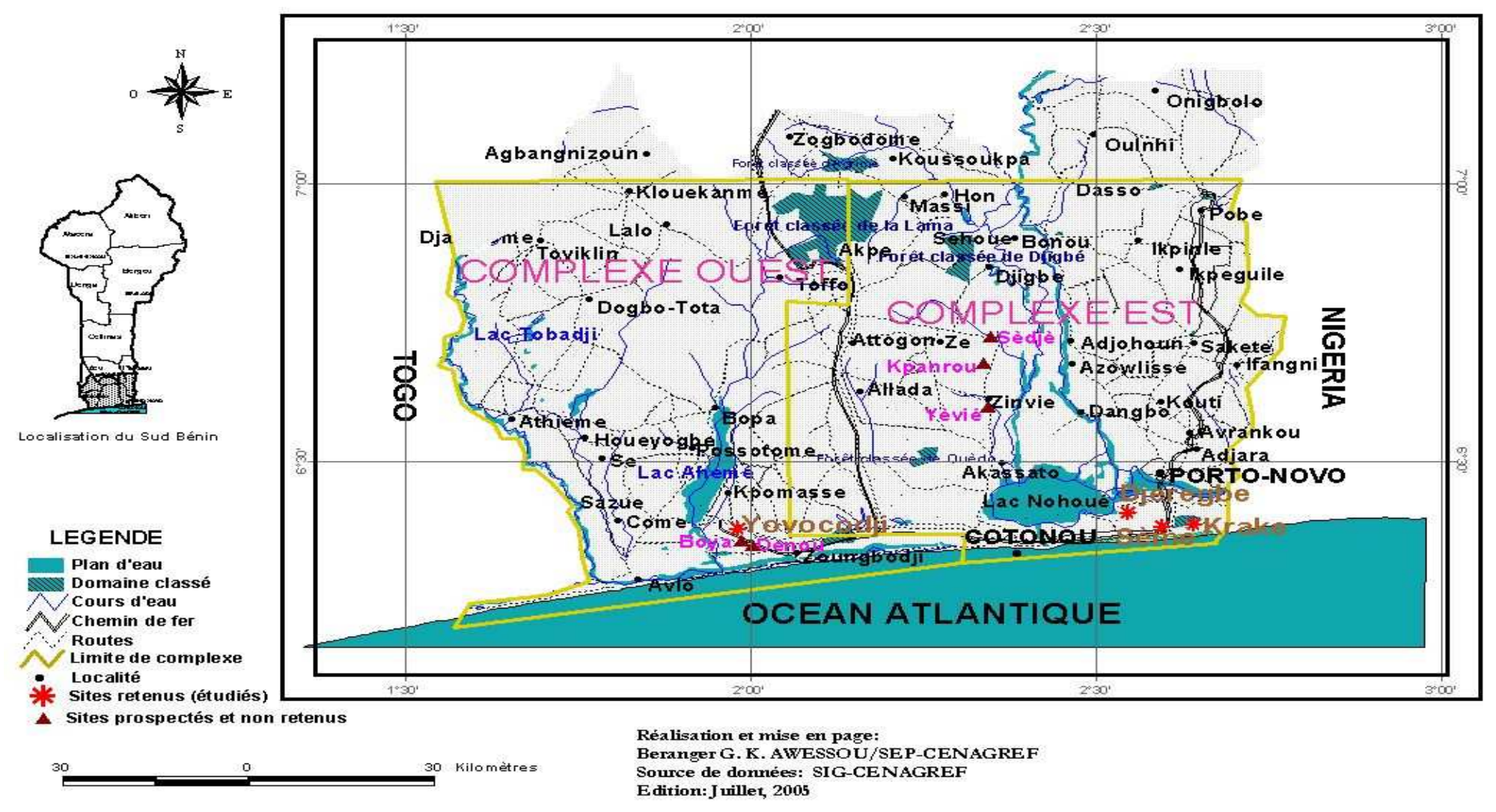

Figure 1 : Zone d'étude présentant les sites prospectés non retenus (triangle) et les sites retenus (étoile) où les données ont été collectées. 

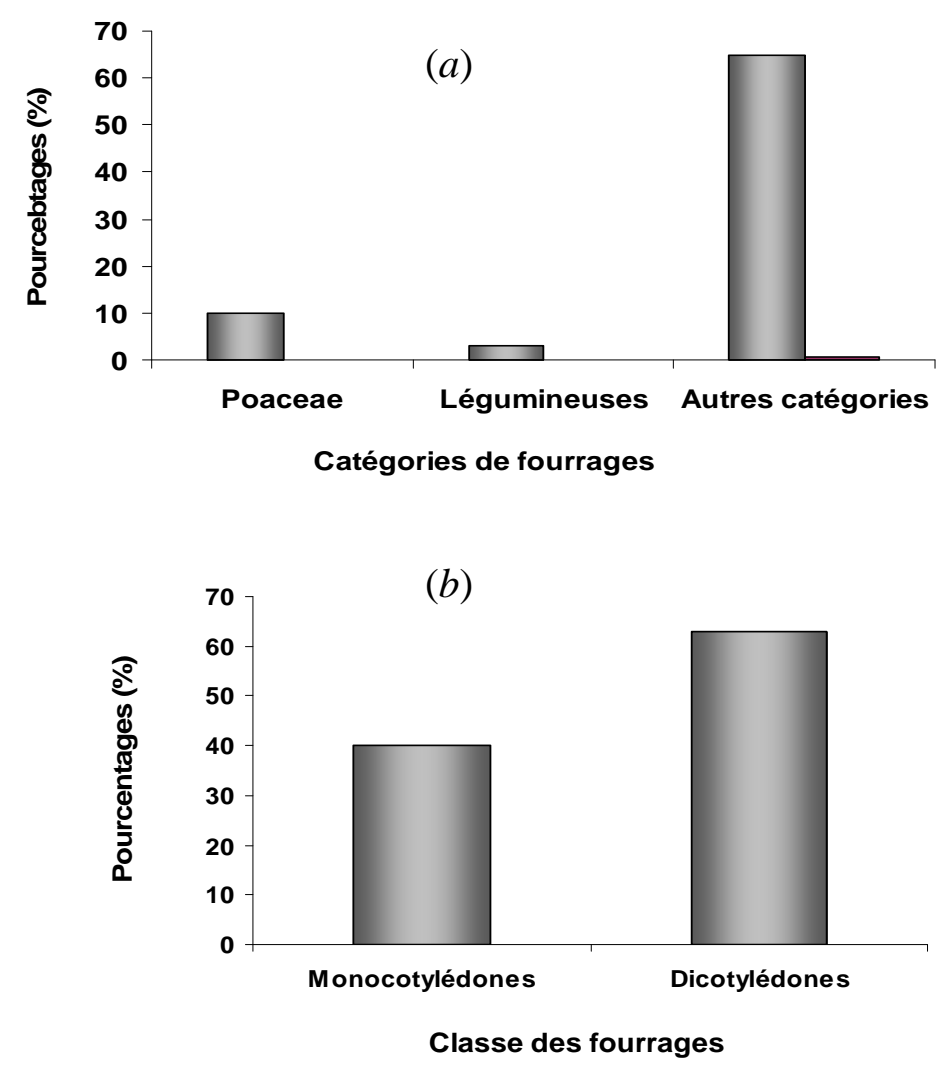

Figure 2 : Proportion des catégories $(\boldsymbol{a})$ et de la classe $(\boldsymbol{b})$ des fourrages dans le régime alimentaire du Sitatunga.

\section{DISCUSSION}

\section{Pâturages exploités par le Sitatunga}

Les prairies marécageuses, les forêts marécageuses, les galeries forestières, les îlots de végétation autour des marécages, les champs et jachères sont les pâturages les plus exploités par le Sitatunga comme Estes (1991) et Nowak (1991) l'avaient déjà rapporté. Pendant les périodes de basses eaux, les zones à inondation permanente ou prolongée sont les pâturages les plus exploités. Par contre, pendant la période de hautes eaux, le Sitatunga exploite les zones à inondation temporaire ou les zones exondées que constituent les îlots de végétation autour des marécages, les champs et les jachères. Ceci est certainement dû au fait que cet animal évite les grands plans d'eau où des courants d'eau pourraient l'entraîner. Il est aussi connu pour sa fidélité qui l'amène à exploiter un même pâturage pendant plusieurs jours de suite (Tweheyo et al., 2000) laissant ainsi des indices de présence qui ont été utilisés au cours de la présente étude pour identifier les pâturages qu'il exploite.

\section{Composition floristique du régime alimentaire du Sitatunga}

$\mathrm{Au}$ cours de cette étude, des méthodes indirectes ont été surtout utilisées. Parmi elles, on peut citer l'analyse micrographique des crottes qui apparaît comme la plus fiable, même si elle ne prend en compte que les feuilles et exclut toutes les autres parties de la plante (fruits, écorces, fleurs, etc.). La limite de cette méthode réside dans le fait qu'on n'est pas toujours sûr d'avoir répertorié dans la collection de référence toutes les espèces 
consommées d'une part, d'autre part les cuticules changent souvent de forme avec le temps, ce qui rend l'identification par comparaison parfois difficile pour l'espèce végétale considérée (Schockert, 1997; Ayégnon, 2004). L'analyse micrographique des caractères épidermiques permet, dans la plupart des cas, de différencier les espèces. La différenciation est plus facile quand il s'agit des classes de végétaux. Mais là encore, certains épidermes des dicotylédones ont des structures complexes qui sont différentes de celles qu'on leur reconnaît, ce qui complique un peu la tâche (Ayégnon, 2004). La capacité d'analyse du chercheur est donc nécessaire pour minimiser les risques d'erreur (Diallo, 1991; Ayégnon, 2002). Malgré ces limites, la méthode micrographique demeure la meilleure et permet de moins perturber l'animal dans son milieu naturel (GarciaGonzalez, 1984 ; Butet, 1987).

L'alimentation des ongulés varie beaucoup d'un ongulé à un autre (Peters, 1983; Bousquet, 1992; Wilmshurst et al., 2000) et en fonction de la disponibilité et de la qualité de la nourriture et surtout en fonction des saisons (Ayégnon, 2004). Mais ceci ne paraît pas évident chez le Sitatunga qui est un ongulé dont le régime alimentaire semble être le même pendant toute les périodes de l'année. Ceci se confirme par la grande similitude observée dans le régime alimentaire pendant la saison sèche et la saison des pluies (Indice de Jaccard, $\mathrm{I}_{\mathrm{J}}=91 \% \geq 50 \%$ ). La stabilité apparente dans le régime alimentaire qui est certainement lié au type d'habitat, les milieux humides, qui ne connaissent pas beaucoup de variations phénologiques du fait de la présence quasi permanente de l'eau, semble expliquer la composition presque identique dudit régime alimentaire. Le Sitatunga a donc un comportement alimentaire non saisonnier contrairement à ce qu'on observe chez la plupart des ongulés des terres fermes (Kassa et al., 2007).

L'un des objectifs spécifiques de ce travail est de déterminer les espèces végétales appétées par le Sitatunga dans un contexte où les pressions anthropiques s'exercent de nos jours sur son habitat. Il est donc heureux que le Sitatunga consomme une gamme très variée d'espèces végétales (Tweheyo et al., 2010) car cette aptitude le rend plus apte à résister dans des habitats perturbés. Les menaces qui pèsent sur les milieux humides sont de plus en plus fortes du simple fait du rythme élevé de réduction du couvert végétal en général mais en ce qui concerne les milieux humides il faut ajouter le comblement des cours d'eau alimentant lesdites zones humides. Ce qui va à terme modifier les caractéristiques des habitats qui servent de refuge aux quelques individus de Sitatunga encore présents au Bénin. Ce à quoi il faut parer dans l'immédiat car si les conditions du milieu sont favorables les Sitatunga sont capables d'y rester en permanence (Magliocca et Gautier-Hion, 2001). La conservation du Sitatunga, une espèce parapluie permettra de conserver d'autres espèces qui sont inféodées à ces milieux aquatiques. La préoccupation majeure à ce niveau est que les zones humides où se retrouve le Sitatunga de nos jours au Bénin, se situent dans la partie méridionale du pays où se concentre la plus forte densité humaine et où il n'existe aucune aire protégée. Ceci interpelle les autorités en charge de l'environnement mais aussi et surtout de la gestion des ressources naturelles. Des décisions doivent être prises au plus haut niveau afin que des actions pertinentes soient menées pour parer au plus pressé dans le court terme mais surtout pour créer dans le moyen et le long termes au moins quelques aires protégées avec des corridors entre elles afin de conserver ces écosystèmes ainsi que leurs biodiversités pour les générations présentes et futures.

\section{Conclusion}

$\mathrm{Au}$ terme de cette étude, on peut retenir que les prairies et les forêts marécageuses sont les pâturages les plus exploités par le Sitatunga et qu'il existe une flexibilité dans l'exploitation de son domaine vital. Enfin, il existe une grande similitude entre le régime alimentaire identifié pendant la saison sèche et la saison pluvieuse. En considérant les 
différentes catégories fourragères, on s'aperçoit que toutes les catégories fourragères sont exploitées rendant ainsi le Sitatunga capable de résister aux perturbations mineures dans son habitat.

\section{REFERENCES}

Adjakpa J. 2001. Inventaire et caractérisation des écosystèmes humides des deux complexes Est et Ouest du sud.

Adomou AC. 2005. Vegetation patterns and environmental gradients in Bénin: Implications for biogeography and conservation. PhD-Thesis, University of Wageningen, p.136.

Akoegninou A. 1984. Contribution à l'étude botanique des îlots de forêt humide semidécidue en République Populaire du Bénin. Thèse de 3è cycle, Univ. Bordeaux III, 250p.

Alfa Gambari A. 2003. Contribution à l'écologie du sitatunga (Tragelaphus speckei) dans la vallée de l'ouémé. Mémoire de fin d'étude, UAC/CPUDAPE. $52 \mathrm{p}+$ annexes.

Ayégnon DTD. 2004. Ecologie alimentaire de quelques grands herbivores du Parc National du W et leur rôle dans la dissémination des herbacées. Mémoire de DEA, Ecole Doctorale Pluridisciplinaire/FLASH/UAC, Bénin, p. 118.

Baumgartner LL, AC Martin. 1939. Plant histology as an aid in squirrel foodhabitat studies. Journal of Wildlife Management, 3: 266-268.

Bousquet B. 1992. Guide des Parcs Nationaux d'Afrique: Afrique du Nord, Afrique de l'Ouest. Paris/France. p. 368.

Butet A. 1987. L'analyse microscopique des fèces: une technique non perturbante d'étude des régimes alimentaires des mammifères phytophages. AVICOLA, 4(1): 36-38.

Chapuis JL. 1980. Méthodes d'étude du régime alimentaire du lapin de garenne Oryctolagus cuniculus (L.) par l'analyse micrographique des fèces. Rev. Ecol., 34: 159-197.
Estes R. 1991. The Behavior Guide to African Mammals. University of California Press: Berkeley and Los Angeles.

Garcia-Gonzalez R. 1984. L'emploi des épidermes végétaux dans la détermination du régime alimentaire de l'Isard dans les Pyrénées occidentales. Colloque International « Ecologie et Biogéographie des Milieux Montagnards et de Haute Altitude » Gabas, 10-12 septembre. Documents d'Écologie Pyrénéenne, IIIIV: $307-313$.

Hanson W B, Sanatani S. 1973. Large Ni Gradients Below the Equatorial $F$ Peak. $J$. Geophys. Res.,78: 1167.

Hessou C, Houngnon. 1999. La faune non aviaire des zones humides du Sud Bénin. PAZH. P.31.

Houndagba J, Tchibozo F, Gnélé J. 2001. Inventaire et caractérisation des écosystèmes humides des complexes Est et Ouest des zones humides du sud Bénin. Rapport de synthèse. PAZH/Cotonou/ Bénin. 71 p.

Johnson MD, Sherry TW. 2001. Effects of food availability on the distribution of migratory warblers among habitats in Jamaica. Journal of Animal Ecology, 70: 546-560.

Kassa B, Libois R, Sinsin B. 2007. Diet and food preference of the waterbuck (Kobus ellipsiprymnus defassa) in the Pendjari National Park, Benin. Afr. J. Ecol., 46: 303-310.

Kidjo FC, Guédou R. 2001. Etudes des potentialités en faune mammalienne et plan de gestion des quelques sites retenus pour abriter le réseau de réserves biologiques dans les zones humides du Sud-Bénin.PAZH/Bénin. p. 61.

Kiley M. 1966. A preliminary investigation into the feeding habits of the waterbuck by faecal analysis. E. Afri. Wildl. J., 4: 153-157.

Kingdon J. 1997. The Kingdon Field Guide to African Mammals. Academic Press: London and New York.

Magliocca F, Gautier-Hion A. 2001. Les clairières en forêt tropicale : des aires à 
protéger en toute priorité. CANOPEE $\mathrm{n}^{\circ}$ 20 - juin 2001.p. 9.

Maizeret C, Boutin JM, Sempere. 1986. Intérêt de la méthode micrographique d'analyse des fècès pour l'étude du régime alimentaire du Chevreuil (Capreolus capreolus). Gibier Faune Sauvage, 3: 159-189.

Maizeret C, Boutin JM, Sempere A. 1986. Intérêt de la méthode micrographique d'analyse des fèces pour l'étude du régime alimentaire du chevreuil (Capreolus capreolou). Gibier et Faune Sauvage, 3: 159-183.

Moegenburg SM, Levey DJ. 2003. Do Frugivores Respond to Fruit Harvest? an Experimental Study of Short-term Responses. Ecology, 84(10): 2600-2612.

Nowak R. 1991. Walker's Mammals of the World. Johns Hopkins University Press: Baltimore, Md.

Peters RH. 1983. The Ecological Implications of Body Size. Cambridge University Press: Cambridge.

Planton L. 1987. Détermination de la composition botanique par analyse microhistologique des épidermes présents dans des collectes du Berger des bols oesophagiens et des fecès. IEMVTCIRAD, p. 29.
Schockert V. 1997. Application de la technique du radio pistage à l'Etude de l'utilisation du temps et de l'espace par le cerf (Elaphecerus elaphus hippelaphus L.) en forêt de saint-Michel et Freyr en fonction de la disponibilité des ressources alimentaires. Mémoire de DESS en science, Université de Liège, p. 56.

Short J. 1981. Diet and feeding behaviour of the forest elephant. Mammalia, 45(2)

Sokpon N, Adjakidjè V. 2001. Caractérisation de la flore et de la végétation des complexes Est et Ouest des zones humides PAZH-Bénin. p33.

Tweheyo M, Amanya BK, Turyahabwe N. 2010. Feeding patterns of sitatunga (Tragelaphus Specki) in the RushebeyaKanyabaha wetland, south western Uganda. African Journal of Ecology, 48: 1045-1052.

Wilmshurst JF, Fryxell JM, Bergman CM. 2000. The allometry of patch selection in ruminants. Proceedings of the Royal Society. Biological Sciences, 267: 345349. 